\title{
FENÓMENOS MIGRATORIOS Y SU INFLUENCIA EN LOS CUIDADOS TRANSCULTURALES EN EL MARCO HOSPITALARIO
}

\author{
Miguel Ángel Fernández Molina
}

Doctor por la U niversidad de Alicante. Licenciado en Antropología Diplomado en Enfermería. Profesor Asociado de la U niversidad de Alicante

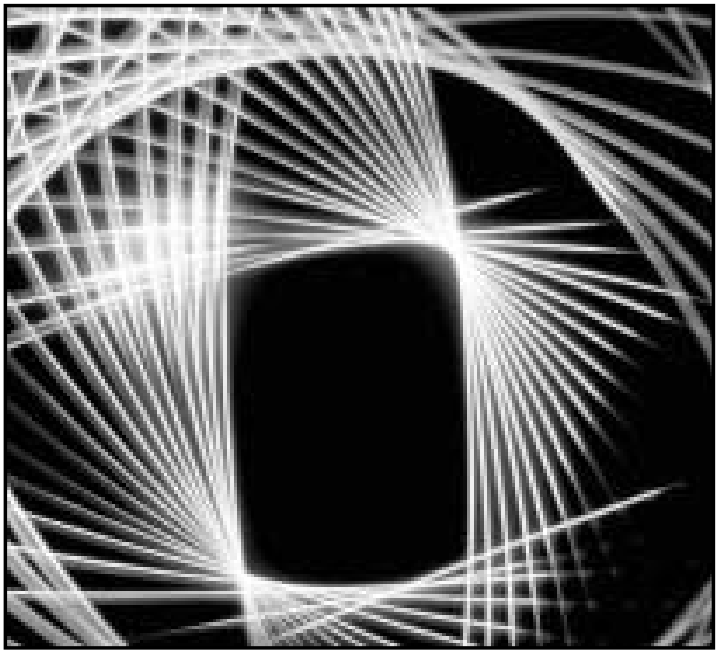

MIGRATION PHENOMENA AND THEIR INFLUENCE IN TRANS-CULTURAL CARE WITHIN A HOSPITAL FRAME

\section{SUMMARY}

$\mathrm{M}$ igratory phenomena and their influence in trans-cultural care within the premises of a Spanish General Hospital are approached in the present study. $8.7 \%$ of the population registered in Spain is foreigner being Alicante the autonomy with the highest rate (19\%). The close relationship between Nursing and Anthropology has proved useful to identify cultural differences among foreign patients admitted to hospital and, as a result, useful tools have furnished the hospital with regards to the most appropriate ways to respectfully handling and treating those various cultural differences. Availability of means is also analyzed and some interesting outcomes and recommendations have been reached by those professionals who are eager to acquire proficiency in cultural fields.
Key Words: anthropology, nursing, foreign population, health, illness, migration

\section{RESUMEN}

Los fenómenos migratorios y su influencia en los cuidados transculturales en el marco de un Hospital General español se abordan en este estudio. El 8,7 \% de la población empadronada en España es extranjera, y Alicante es la provincia con la mayor proporción (19 \%). El maridaje de Enfermería y Antropología ha sido utilizado para identificar las diferencias culturales entre los pacientes extranjeros ingresados y, como resultado, se facilita una útil herramienta consistente en una guía para el respeto a la diversidad cultural. Los recursos disponibles son analizados y se alcanzan algunas conclusiones y recomendaciones interesantes así como el hallazgo entre los profesionales de un profundo deseo de adquirir competencia cultural.

Palabras clave: Antropología, enfermería, extranjeros, salud, enfermedad, migración

\section{INTRODUCCIÓN}

En un contexto donde se viene observando una creciente preocupación social por el probable impacto de la inmigración en el sector sanitario, se hacen imprescindibles estudios que se aproximen a los hechos de forma que puedan objetivar esta fenomenología, ofreciendo una visión nítida, desprovista de prejuicios, que concrete de forma cuantitativa y cualitativa la dimensión exacta de una nueva sociedad más diversa en lo cultural, con tradiciones, creencias y prácticas que se manifiestan en aspectos referidos a la salud y la enfermedad.

Las migraciones han sido una constante en el curso de la evolución humana, y la especie humana 
ha demostrado a lo largo de su historia una extraordinaria capacidad de adaptación a nuevos entornos. Actualmente, las poblaciones siguen migrando, las circunstancias por las que se mueven son tan variadas como las culturas de las cuales proceden, pero hay un hecho en la migración que es universal, "los inmigrantes llevan consigo algo más que maletas" (KEMP y RASBRIDGE, 2004).

Para muchos occidentales, desde el punto de vista sanitario, los inmigrantes de todo tipo (y refugiados, en su caso) portan diversos perfiles epidemiológicos propios y enfermedades endémicas de sus lugares de origen. Además, la amplia experiencia pasada del inmigrante contrasta enormemente con la medicina alopática o científica. Pero lo más importante de todo es que, los extranjeros en general, traen con ellos sus creencias culturales y prácticas, incluidas aquellas que tienen que ver con la salud y la enfermedad, y que con frecuencia pueden llegar a chocar con las normas del país anfitrión. El estudio de la salud entre los extranjeros es realmente un estudio de la cultura, una competencia de la Antropología, porque mientras que la enfermedad puede ser universal, la definición de salud, la interpretación de los síntomas, los remedios y tratamientos para promocionar y recuperar la salud están definidos culturalmente.

Por estas razones, los estudios sobre los cuidados aplicados a pacientes extranjeros ingresados en hospitales desde la perspectiva de la Enfermería Transcultural se justifica por los acentuados cambios demográficos que está experimentando la sociedad española -y más concretamente en determinadas regiones- por sus posibles repercusiones en materia de salud, enfermedad y cuidados. A 1 de enero de $2006^{1}$-sobre una población de 44.395.300 habitantes- los extranjeros empadronados suponían en el conjunto del Estado Español un 8,7\% (3.884.600), con una variación interanual del $4,13 \%$ frente al $0,65 \%$ de la población total. En la Comunidad Valenciana un $13,4 \%$ de sus empadronados son extranjeros (641.000) y Alicante ya supera los $321.640(18,56 \%)$ de enero 2005. Ciertamente, la situación geográfica y económica de España la convierte en uno de los principales destinos de los movimientos migratorios, siendo las comunidades con mayor porcentaje de extranjeros Cataluña (866.800 y 12,2\%), Madrid (695.600 y $11,8 \%)$, Comunidad Valenciana (641.000 y 13,4\%), Región de Murcia (181.800 y 13,3\%) e Islas Baleares (153.700 y 15,6\%). Los extranjeros en España han pasado de representar el $1.6 \%$ de la población en el año 1998 al $8.7 \%$ actual, siendo los marroquíes el colectivo con mayor presencia en el país seguidos de cerca por los ecuatorianos y después por los rumanos, colombianos y británicos (INE, 2006).

Desde la Antropología (análisis y descripción de las culturas) y la Enfermería (estudio de los cuidados) se pueden investigar unos hechos -que bien podríamos calificar de "etnográficos"- en relación con la diversidad cultural y la enfermedad en el contexto de nuestros centros sanitarios, como es la utilización de los servicios de salud por parte de pacientes extranjeros y su necesidad de cuidados. Entre el personal sanitario se percibe un aumento en el número de pacientes de origen extranjero (inmigrantes o no) que acuden a los hospitales públicos y centros de Atención Primaria, lo que está dando lugar -aunque de forma aislada- a la aparición de determinadas actitudes (se les percibe como personas sin derechos a las que se les da atención gratuitamente), prejuicios, estereotipos, comentarios de carácter etnocéntrico o, peor aún, suscitan el tópico generalizado de que "consumen el presupuesto en Sanidad de los españoles".

\section{ATENCIÓN A PACIENTES EXTRANJEROS EN HOSPITALES Y CENTROS ESPAÑOLES}

Debemos partir de una constatación, y es que los cuidados están polarizados sobre una sola cultura: la occidental. Además, la cultura hospitalaria es muy etnocéntrica (LEININGER, 1995). El etnocentrismo consiste en rechazar las formas de pensar, los valores, las creencias de otras sociedades y considerar las propias como superiores. El hospital tiene valores que considera superiores y a imponer a los demás (ROHRBACH, 1998).

Cuando se diseñaron las organizaciones sanitarias de nuestro país tal y como hoy las conocemos, nadie podía llegar a vislumbrar la posibilidad de coexistencia en el seno de una misma institución de 
diferentes tradiciones y practicas culturales. Tampoco en el pasado, ni autoridades sanitarias ni los propios profesionales tuvieron que afrontar los dilemas que plantea la libertad cultural, entre ellos si es moralmente permisible (de acuerdo con los principios liberales) imponer a los extranjeros unas expectativas de comportamiento cimentadas en el modo de actuar de la mayoría (MENDEZ, 2004).

Dada la relativa novedad de la preocupación por la asistencia a pacientes extranjeros en nuestros centros, no existen aún muchos estudios que aborden específicamente este fenómeno. Algunos trabajos científicos de autores nacionales analizan los problemas de salud generales y particulares que presenta el colectivo de inmigrantes. Se han hallado varios trabajos en el campo de la tuberculosis importada, VIH, salud mental, atención maternoinfantil, trastornos psiquiátricos y otras enfermedades infecciosas (TORRÚS, 2006). En cuanto al VIH también se ha detectado que el porcentaje de inmigrantes afectados podría ir en aumento, ya que una parte de la población inmigrante adquirirá la infección en nuestro país debido al uso de la prostitución (GASCÓN, 2003).

Desde el puno de vista clínico, el estudio del uso de nuestros hospitales por parte de la población inmigrante es un fenómeno reciente, con pocos precedentes. Pueden citarse trabajos como el realizado en el Hospital de Mataró (Barcelona) acerca de enfermedades importadas en inmigrantes africanos: estudio de 1.321 pacientes (ROCA et al, 2002), o el trabajo realizado en hospitales públicos de Valencia sobre un total de 248 altas de inmigrantes (SALAZAR et al, 2003) en un intento de aportar información sobre la utilización de servicios hospitalarios así como cuantificar de modo objetivo las patologías más urgentes y comprometedoras. Una de sus conclusiones más llamativas en este último era que la proporción de enfermedades infecciosas y, dentro de ellas, el número de infecto-contagiosas no contribuían a alimentar las opiniones difundidas sobre importación o reemergencia de enfermedades desde los colectivos de inmigrantes.

Cuando un extranjero ingresa como paciente en uno de nuestros hospitales, entra en contacto con lo que podríamos definir como "la cultura hospitalaria”, donde las/los enfermeras/os y médicos actúan en base a sus creencias, valores, cultura, etc., y creen que deben actuar así por el bien del paciente y su familia, ya que son ellos los profesionales de la salud. Esta actitud etnocéntrica es un sesgo muy frecuente dentro de la actuación del personal, pero quizás la situación se agudice más en el caso de pacientes con una herencia cultural marcadamente diferente al del "cuidador". Estudios realizados en Suecia para identificar diversidades y similitudes en los cuidados y valores culturales entre enfermeras suecas y enfermeras iraníes en un hospital de Estocolmo (CONTE, 2004) son muestra de la necesidad de tener en cuenta no sólo la herencia cultural del paciente, sino la del propio profesional.

Aunque tímidamente, comienza a detectarse una preocupación en nuestro contexto por evaluar la confianza que tienen las enfermeras españolas a la hora de atender a pacientes de grupos culturales diversos y así desarrollar programas de formación pre-grado que contemplen la administración de cuidados culturalmente sensibles (VARGAS et al., 2004).

Desde la perspectiva del paciente, la situación actual más común es que este ingrese junto con su familia, en un lugar donde pierden el control de la situación, que pasa a manos de los profesionales que ahí trabajan, se plantean problemas de índole lingüístico y cultural ligados a hábitos e incluso a la concepción de la prestación sanitaria que se les ofrece (LÓPEZ y LORENZO, 2004). La habitación es en la mayoría de veces compartida con otro paciente que puede llegar a simbolizar la "alteridad" en toda su plenitud; se imponen unos horarios de comida, medicación, descanso, etc., que no se corresponden con los habituales del paciente en su domicilio. Además, las enfermeras responsables de su cuidado poseen actitudes diversas ante el sinfín de procesos que aguardan al paciente (procedimientos, cuidados, higiene, información, diagnósticos, pronósticos, dolor y hasta... la muerte). Todo este entramado de valores, creencias, imposiciones, etc., puede provocar una inadecuada aceptación del episodio asistencial por parte del propio paciente y su familia, reflejada en la aparición de un elevado número de insatisfacciones, frustraciones o percepciones negativas de las que pueden o no quedar constancia escrita y que en el peor de los casos pueden llegar al alta voluntaria. 


\section{LA MEDIACIÓN CULTURAL EN EL CON- TEXTO SANITARIO}

Cuando, en un contexto pluriétnico o multicultural, la presencia de varias lenguas, sistemas de valores y modelos comunicativos provoca que la comunicación entre dos partes no pueda llevarse a cabo sin el puente de una tercera persona, hablamos de mediación. Aunque tímidamente, ha surgido en algunos centros la denominada "mediación intercultural", fenómeno bastante reciente que en España no cuenta con mucho más de 10 años. Se trata de una figura que aún no está del todo definida, ni siquiera regularizada profesionalmente, pues aunque la figura del mediador intercultural ya está reconocida por el Ministerio del Interior (según Real Decreto 638/2000 de 11 de mayo), a efectos prácticos no tiene relevancia en los Hospitales. Actualmente no existe un título oficial de mediador intercultural, ni un sistema de formación y acreditación. La mediación intercultural suele estar relacionada con el ámbito del trabajo social, y las pocas iniciativas formativas en este campo, contando o no con el apoyo académico universitario, se han puesto en marcha desde ONG's y servicios sociales de ayuntamientos (SALES, 2005).

La Mediación Intercultural (o mediación social en contextos como el arriba descrito) se entiende como una modalidad de intervención de terceras partes, en y sobre situaciones sociales de multiculturalidad significativa, orientada hacia la consecución del reconocimiento del "Otro" y el acercamiento de las partes, la comunicación y comprensión mutua, el aprendizaje y desarrollo de la convivencia, la regulación de conflictos y la adecuación institucional, entre actores sociales o institucionales etnoculturalmente diferenciados (GIMÉNEZ, 1997).

Hay publicados estudios sobre los efectos de la mediación intercultural sobre la calidad de la atención prestada (VERREPT y LOUCKX, 1997), en contextos europeos. Según estos, los profesionales sanitarios, pacientes y mediadores culturales confirman que la introducción de la mediación intercultural incrementó de manera significativa la calidad de la atención cuando se utilizaron de manera adecuada sus servicios. Los profesionales participantes en estos estudios manifestaron que el programa de mediación intercultural se debería mantener (donde estuviera implantado) y convertirse en un servicio habitual a disposición de los pacientes de minorías étnicas y del personal sanitario. La mejora más importante de este servicio está asociada al hecho de que las personas mediadoras facilitan el intercambio de información detallada y correcta entre el personal sanitario y los pacientes, contribuyendo a ello la menor inhibición de los pacientes a la hora de relatar su caso en presencia de una mediadora cultural.

Las referencias a este tipo de labor en España se limitan a grupos aislados muy concretos, como la de la Escuela de Mediadores de la Comunidad de Madrid, en colaboración con Cruz Roja, desarrollando el Programa "Migración y Multiculturalidad". Como resultado de la colaboración entre esta Escuela y el Ayuntamiento de Madrid desde 1997 funciona el Servicio de Mediación Social Intercultural, inspirado en sistemas similares de Italia, Suecia o Reino Unido. Otro foco de atención en torno a la mediación intercultural lo constituyen la Federación Andalucía Acoge y el AEP Desenvolupament Comunitari de Cataluña, que han venido colaborando desde 1998 (VV.AA., 2002). En la Comunidad Valenciana se puede citar la experiencia que aporta una Mediadora, Saloua Laghrich, del Servicio de Atención al Inmigrante que depende de la Conselleria de Bienestar Social de la Generalitat Valenciana. Describe unas intervenciones muy puntuales y concretas en el Hospital La Fe de Valencia y que ilustran la eficacia de actuar en situaciones tremendamente difíciles tanto para el paciente y como para el equipo asistencial debido a barreras culturales (LAGHRICH, 2004).

En la práctica, son los profesionales sanitarios de los hospitales (y de Atención Primaria) los que actúan la mayor parte de las veces de Mediadores. Mediar no sólo es traducir las palabras (labor esencial de intérpretes/traductores) sino que va más allá, abarcando todos los aspectos de la comunicación no verbal (olor, gestos, movimientos corporales, silencios, ritos, etc.), que culturalmente son claves. Además de conocimientos lingüísticos, debe tener presentes datos de la sociedad y la cultura a la que pertenece el paciente extranjero: relaciones de parentesco, religión, percepción de lo que significa la salud y la enfermedad, costumbres y aspectos culturales relevantes. Además, debe evitar caer en el estereotipo de considerar al extranjero como un "miembro típico" de su cultura teniendo en cuenta 
la variable personal de cada cual y además la adaptación o cambio que puede devenir del contacto entre culturas.

\section{DISEÑO DEL ESTUDIO}

El estudio que se presenta interrelacionan íntimamente dos disciplinas científicas: por una parte la Enfermería, que ha seguido tradicionalmente una metodología cuantitativa inspirada en el paradigma positivista y, por otra, la Antropología, idónea para una aproximación cualitativa de los fenómenos que rodean a los cuidados enfermeros. Es, pues, descriptivo, comparativo y fenomenológico, siendo el Campo de Estudio: el Hospital General Universitario de Alicante (HGUA), centro de referencia provincial para 1.732 .389 personas siendo el segundo de la Comunidad Valenciana en número de camas (815). En cuanto a la Metodología, se consideró la complementariedad de métodos cuantitativos y cualitativos. Método Cuantitativo: análisis descriptivo retrospectivo de los años 2001 al 2004 de los pacientes extranjeros ingresados; análisis de la información al alta; análisis del Censo 2001 y datos del Padrón 2003, 2004 y 2005 del INE; estudio descriptivo transversal a través de 40 cuestionarios a enfermeras. Método Cualitativo: estudio fenomenológico usando la etnografía y la etnología; aplicación de técnicas como la Observación Directa, Observación Participante, Entrevistas Abiertas, Entrevistas Estructuradas, Historias de Vida, Grupos de Discusión y Análisis de Contenido.

\section{PRESENTACIÓN DE LOS RESULTADOS DEL ESTUDIO EN EL HGUA}

El HGUA fue entre 2001 y 2005 un campo de estudio $^{2}$ de este fenómeno migratorio y su influencia en los cuidados Transculturales. El Objetivo General fue el de identificar la diversidad cultural entre los pacientes de nacionalidad extranjera ingresados en este hospital y la razón fundamental de su elección se sustentó en la creciente presencia de pacientes extranjeros, cuyos valores, creencias, normas y prácticas de salud pueden diferir de aquellos que les debían cuidar: los profesionales sanitarios. Siendo una investigación de carácter antropo- lógica, la multitud de matices que rodearon el proceso asistencial le confirieron un marcado interés cultural, social, económico y naturalmente sanitario. La importancia del tema radica tanto en la necesidad de ofrecer unos cuidados de calidad adaptados a las necesidades de los extranjeros, como en las consecuencias de una supuesta falta de sensibilidad cultural de los cuidadores. La sensibilidad cultural debe ser entendida como el hecho de que los profesionales consideren los factores culturales como relevantes para la salud, enfermedad y a la hora de administrar cuidados (MARTSOLF, 1999). Así, pues, si bien el cuidado es universal, las acciones, expresiones, patrones, estilos de vida y significados de los cuidados podían ser diferentes y el problema era saber si estábamos o no proporcionando cuidados culturalmente congruentes.

Los resultados demográficos de la investigación condujeron a una categorización preliminar con tres grandes grupos de población extranjera que, por su notable presencia en el contexto del estudio, justificó el hacer un análisis etnográfico. A éstos grupos se sumó un cuarto (el Asiático) que, aún no siendo muy significativo en número de ingresos todavía, debido a su aumento creciente en España y la diferencia en herencia cultural sobre la salud y la enfermedad, se incluyó:

1) Población procedente de Países Árabes del Oriente Medio y Norte de África

2) Población procedente de Países de habla Hispana (no españoles)

3) Población procedente de Países Nórdicos y Centroeuropeos.

4) Población procedente de Países Asiáticos

En el período que transcurre entre el 1 de enero de 2001 y 31 de diciembre de 2004 ingresaron 9792 pacientes extranjeros, de los cuales 6224 pertenecían a uno de los 4 grupos diana, 2320 pacientes ingresaron sin que el programa IRIS ${ }^{\circledR}$ les asignara un descriptor de país de nacimiento (por tanto se puede afirmar que un número indeterminado podría pertenecer a alguno de los grupos diana) y, finalmente, 1248 pacientes eran de otros países.

Como cuestión preliminar antes de proceder a exponer los frutos de este estudio etnográfico (que

\footnotetext{
${ }^{2}$ Tesis Doctoral de Miguel Ángel Fernández Molina "Estudio de los cuidados a pacientes extranjeros ingresados en el HGUA desde la perspectiva de la Enfermería Transcultural" Universidad de Alicante Noviembre 2005.
} 
también podría denominarse "etnoenfermero"), debe tenerse presente lo que afirma Mulhall quien considera a los Antropólogos culpables de etiquetas culturales (MULHALL, 1994). Ciertamente, estudios sobre el dolor en los diferentes grupos étnicos han sugerido que, por ejemplo, los Italianos son hipocondríacos, que requieren poca información sobre su tratamiento y no se espera de ellos que sean puntuales a sus citas médicas ni que se adhieran a su terapia. Este etiquetado universal queda desmentido en estudios más profundos que indican que las creencias y prácticas en relación con el dolor difieren fundamentalmente entre grupos, y que tales generalizaciones están basadas en la visión particular del propio autor. Es intención del presente investigador no imponer las propias categorías de significado sobre los pacientes observados así como no perpetuar ciertas imágenes estereotipadas del "otro".

Podemos afirmar que la población extranjera a estudiar corresponde a dos grupos bien diferenciados:

1. Los extranjeros comunitarios. Residentes y turistas de países de la Unión Europea.

2. Los extranjeros no comunitarios: inmigrantes regularizados y no regularizados, sobre todo del Magreb (Marruecos / Argelia), Latinoamérica y Asía.

Se realizó una investigación etnográfica mediante una descripción de los eventos que tienen lugar en la vida de los grupos escogidos, con especial consideración de las estructuras sociales y la conducta de los pacientes y familiares como miembros del grupo, así como de sus interpretaciones y significados de la cultura a la que pertenecen. Se pretendió describir a los grupos de extranjeros en profundidad -aunque no en su ámbito natural- y comprenderlos desde el punto de vista de quienes estamos implicados con ellos (los profesionales sanitarios). Mediante análisis etnográfico y a través de las variables que a continuación se presentan, se intentó plasmar las creencias y prácticas que sobre la salud influyen derivado del origen étnico y cultural de las poblaciones señaladas con anterioridad y que se dan en el lugar de la investigación:

- Religión

- Prácticas de salud

- Costumbres sociales

- Salud maternal e infantil
- Intimidad

- Dieta

De los resultados del citado estudio (FERNÁNDEZ, 2006) -a modo de resumen- destacar:

- 1. Se evidenció la existencia en el HGUA de una diversidad cultural significativa y creciente entre sus pacientes, detectándose cuidados humanos diversos (diferentes) y universales (similares) en los extranjeros.

- 2. En el 2004, el 7.75\% de los ingresos correspondieron a extranjeros y entre enero 2001 y junio 2004 se contabilizaron 8.172 ingresos (correspondientes a 5.794 pacientes) de 91 nacionalidades en 57 servicios, con una edad media de 42.69 años; el $49 \%$ eran extranjeros no comunitarios y un $25 \%$ eran residentes o turistas de la Unión Europea. Los países con más ingresos fueron Marruecos (10.4\% de los extranjeros), Colombia (8.5\%), Francia (8.0\%) Ecuador (7.9\%) y Reino Unido (6.9\%). El Servicio Médico con mas ingresos fue Obstetricia (17.49\% de los extranjeros) seguido de Ginecología $(8.11 \%)$-sumando ambos un $25.6 \%$ de todos los casos- seguido de Cirugía General (6.16\%), Unidad de Corta Estancia (5.80\%), Cirugía Ortopédica y Traumatología (3.89\%) hasta 57 Servicios Médicos y Quirúrgicos.

- 3. A pesar de que el $18.5 \%$ de la población de la provincia de Alicante era extranjera, sólo ingresó un $7.75 \%$, lo que cuestiona la percepción de que los inmigrantes copan los recursos hospitalarios; tampoco son portadores de enfermedades infectocontagiosas graves, raras e incluso erradicadas que precisen hospitalización ya que la casuística (GRD's) encontrada no se orienta en ese sentido. El turista o residente europeo está dando lugar a un fenómeno nuevo que se denomina "turismo sanitario", que acarrea unos costes muy superiores al gasto en el que incurre el extranjero inmigrante en busca de trabajo. Además, los extranjeros son donantes de órganos generosos ya que el $37 \%$ de las donaciones en 2005 fueron de extranjeros y su tasa de negativas familiares fue de $0 \%$.

- 4. Los pacientes extranjeros conservan su herencia cultural durante el ingreso y viven el episodio asistencial de manera distinta en algunos casos según demuestra el estudio etnoenfermero llevado a cabo. 
- 5. El HGUA cuenta con recursos específicos para extranjeros que pueden ser ampliados.

- 6. Los profesionales de enfermería son conscientes de las diferencias culturales.

- 7. Se van incorporando aspectos culturales en el Proceso de Enfermería.

- 8. Se detectó la necesidad de formar a los profesionales en habilidades que permitan preservar, acomodar o remodelar los cuidados de sus pacientes extranjeros, y manifiestan su deseo de adquirir competencia cultural.

Asimismo, los profesionales propusieron recomendaciones y sugerencias para mejorar el cuidado a los pacientes extranjeros, conscientes de un fenómeno creciente como es la diversidad cultural en la sociedad y en los centros sanitarios.

Finalmente, añadir como contribución personal que estamos ante una fenomenología que interesa, en mayor o menor medida, tanto al personal asistencial como a los docentes, investigadores y responsables de Administraciones Públicas. Para abordar toda la problemática encontrada, la perspectiva Transcultural se revela como idónea ya que evita prejuicios o malos entendidos a la hora de cuidar pacientes de origen cultural diferente, aporta datos clarificadores que facilitan la planificación de los recursos y, por último, estimula a los profesionales para que adquieran habilidades que ayuden a unos encuentros beneficiosos con sus pacientes extranjeros.

\section{REFERENCIAS BIBLIOGRAFÍCAS}

- CONTE, H. (2004) Identifying diversities and similarities in profesional cultural caring and care values in Swedish and Iranian nurses in Stokholm using the Critical Incident Technique, Alicante, III Jornadas Internacionales de Cultura de los Cuidados \& 30th Annual Conference of the Transcultural Nursing Society y VI Reunión sobre Investigación Cualitativa en Salud, 14-16 junio 2004, Libro de Abstracts, (Póster).

- FERNÁNDEZ MOLINA, M.A. (2006) Antropología de los Cuidados. Estudio de los cuidados a pacientes extranjeros hospitalizados desde la perspectiva de la Enfermería Transcultural. Edita: Consejo de Enfermería de la Comunidad Valenciana (CECOVA), Alicante.

- GASCÓN, J. (2003) Enfermedades infecciosas e inmigración, Enfermedades Infecciosas Microbiología Clínica 2003; 21 (10): 535-9

- GIMENEZ ROMERO, C. (1997) "La naturaleza de la mediación intercultural", Revista de Migraciones, 2, pp. 125-159

- INSTITUTO NACIONAL DE ESTADÍSTICA (INE) Explotación Estadística del Padrón Municipal a 1 de enero de 2005. Publicado el 17 de enero de 2006. Disponible en http://www.ine.es
- KEMP, C y RASBRIDGE, L.A. (2004) Refugee and immigrant health. A handbook for health professionals. Ed. Cambridge University Press, Cambridge, England.

- LAGHRICH, S. (2004) Reflexiones sobre la mediación intercultural y experiencias desde la Comunidad Valenciana. Tonos, Revista Electrónica de Estudios Filológicos, $\mathrm{N}^{\mathrm{o}} 8$, Diciembre 2004. (disponible en: www.um.es/tonosdigital)

- LEININGER, M. (1995) Transcultural Nursing. Concepts, Theories, Research and Practices.2nd Ed. McGraw Hill, New York, EE.UU.

- LÓPEZ LLORENS, P.L. y LORENZO GARCÍA, A. (2004) Acogida y atención hospitalaria a extranjeros. Un nuevo reto para enfermería. Alicante, XV Jornadas de Interrelación de Enfermería, pp- 57-66, Edita Consejo de Enfermería de la Comunidad Valenciana, №38 (Comunicación escrita).

- MARTSOLF, D.S. (1999) Cultural aspects of Orthopaedic Nursing. Orthopaedic Nursing, March/April 1999, pp. 65-71.

- MÉNDEZ MÉNDEZ, E. (2004) Políticas públicas de acomodación de los inmigrantes en el ámbito sanitario, Quadern CAPS, 2004, No 32, pp. 18-23.

- MULHALL, A. (1994) Anthropology: a model for nursing, Nursing Standard, April 27, Vol. 8, No 31

- ROCA, C., BALANZÓ, X., FERNÁNDEZ-ROURE, J.L., SAUCA, G., SAVALL, R., GASCÓN, J. y CORACHAN, M. (2002) Enfermedades importadas en inmigrantes africanos: estudio de 1.321 pacientes, Medicina Clínica 2002;119(16):616-9

- ROHRBACH-VIADAS, C. (1998) Introducción a la teoría de los cuidados culturales enfermeros, de la diversidad y de la universalidad de Madeleine Leininger, Alicante, Cultura de los Cuidados 1 Semestre 1998, Año II - No 3. Universidad de Alicante

- SALAZAR, A., NAVARRO-CALDERÓN, E., ALMELA, F., ABAD, I., BORRÁS, R., GONZÁLEZ, A., GOSÁLBEZ, E., PALAU, P., ROMERO, R. SOTO, L., TABERNER, F. (2003) Altas hospitalarias de la población inmigrante en la ciudad de Valencia, Boletín Epidemiológico, Instituto de Salud Carlos III, 2003, vol. 11 n $5 / 49-60$.

- SALES SALVADOR, D. (2005) Panorama de la mediación intercultural y la traducción/interpretación en los servicios públicos en España. Translation Journal. Volume 9, $\mathrm{N}^{\circ} 1$ January 2005. URL: http://accurapid.com/journal/31mediacion.htm

- TORRÚS TENDERO, D. (2006) Problemas de salud en la población inmigrante, Apuntes del Curso de Formación Continua EVES, Hospital General Universitario de Alicante, enero 2006

- VARGAS JIMÉNEZ, J.A., MOLINA CONTRERAS, J.L., SHELLMAN, J. y BERNAL, H. (2004) The confidence of Spanish nurses in caring for culturally diverse groups, Alicante, III Jornadas Internacionales de Cultura de los Cuidados \& 30th Annual Conference of the Transcultural Nursing Society y VI Reunión sobre Investigación Cualitativa en Salud, 14-16 junio 2004, Libro de Abstracts, (Comunicación).

- VERREPT, H. y LOUCKX, F. (1997) Mediadores de salud en el sistema sanitario belga, en Solas,O., Ugalde. (ed.), Inmigración, salud y políticas sociales, Granada: Escuela Andaluza de Salud Pública, 209-230.

- VV.AA. (2002) Mediación intercultural. Una propuesta para la formación. Madrid: Editorial Popular. 